\title{
Polyaniline-wrapping hollow sulfur with MCM-41 template and improved capacity and cycling performance of lithium sulfur batteries
}

Yan ling $\mathrm{An}^{\mathrm{a}}$, Wenlong Song ${ }^{\mathrm{ab} *}$, Pan $\mathrm{Wei}^{\mathrm{a}}$, Meiqiang $\mathrm{Fan}^{\mathrm{ab} *}$, Haichao Chen ${ }^{\mathrm{a}}$, QiangJian Ju', Da Chen ${ }^{\mathrm{a}}$, Guanglei Tian ${ }^{\mathrm{a}}$, ChunJu Lva ${ }^{\mathrm{a}}$ Kangying Shu ${ }^{\mathrm{a}}$

\begin{abstract}
A conductive polyaniline-wrapping hollow sulfur composite used as a cathode material for lithium/sulfur batteries was successfully fabricated by a facile and controllable chemical reaction with MCM-41 template. The polyaniline-wrapping hollow sulfur composite had a good reversible capacity of $506.3 \mathrm{mAh} \mathrm{g}^{-1}$ at the 150 th cycle, reflecting the low loss of sulfur and polysulfide encapsulated by polyaniline during the charge and discharge cycles. The results of SEM and EIS show that the polyaniline-wrapping hollow sulfur composite presented many good electron-conducting paths for the rechargeability of lithium/sulfur battery. XRD and TG-DSC results revealed the formation of fine sulfur particles encapsulated by polyaniline and sulfur distributed on the polyaniline surface because of low sulfur melting temperature, which was the key reason for the activation.
\end{abstract}

Keywords: lithium/sulfur battery; polyaniline wrapping hollow sulfur composite; 
conductive polymer; MCM-41

\section{Introduction}

Energy storage is a key factor related to sustainable development worldwide. Lithium ion battery is a new and attractive green energy storage device, which has developed rapidly over the last decade. This battery is a good choice for mobile phones, notebook computers, portable electric tools, and electric vehicles because it possesses high specific capacity, long cycle, and no environmental pollution, among others. However, conventional lithium ion battery still has low energy density and high cost, which prevent wide application in electric vehicles. It is the key factor to develop high-performance and low-cost materials and fabrication technologies for lithium ion battery. Compared with the other components of lithium ion battery, the cathode electrode material is the key component that affects electrochemical performance, safety, and cost of the entire cell. $\mathrm{LiFePO}_{4}, \mathrm{LiCoO}_{2}$, and $\mathrm{LiMn}_{2} \mathrm{O}_{4}$ are widely used cathode materials for commercial lithium ion battery. These cathode materials have low theoretical capacity and cannot meet the growing energy density requirement of batteries. Sulfur-based materials are a promising cathode; sulfur has many advantages, such as high theoretical capacity $\left(1672 \mathrm{mAh} \mathrm{g}^{-1}\right)$, low cost and abundant reserves [1-3], compared to conventional lithium-ion batteries and other emerging lithium-based batteries. However, several difficulties exist for sulfur application in lithium ion batteries because sulfur has an insulating nature, large volume expansion, and dissoluble polysulfide species formed in the discharge process $[4,5]$. These shortcomings lead to capacity fading, Coulombic efficiency decrease, 
and low C-rate because of the loss of active materials and the side reactions on the lithium metal anode. Resolving these shortcomings is necessary to stimulate the development of sulfur cathode.

Sulfur particles limited inside the conductive polymers is a good method for improving the electrochemical performance of sulfur cathode. A polymer presents a flexible nanostructure and contains a large void space inside the polymer shell, which can accommodate the volume expansion of sulfur and decrease polysulfide dissolution during lithiation [6]. The sulfur cathode coated or modified with electro-polymerized conductive polyaniline (PANI) and polyprrole had improved the electrochemical performance of rechargeable batteries [7, 8]. Qiu [9] co-heated the mixture of pyrrole-co-aniline (PPyA) and sublimed sulfur at $160^{\circ} \mathrm{C}$ for $24 \mathrm{~h}$, where the sulfur was limited inside the nanofiber network structure of PPyA. The nano-dispersed composite exhibited an initial specific capacity of up to $1285 \mathrm{mAh} \mathrm{g}^{-1}$ and remained at $866 \mathrm{mAh} \mathrm{g}^{-1}$ after 40 cycles. Xiao L. prepared a vulcanized polyaniline nanotube/sulfur composite by heating a mixture of polyaniline nanotube and sulfur at $280{ }^{\circ} \mathrm{C}$. The composite presented a discharge capacity of $837 \mathrm{mAh} \mathrm{g}^{-1}$ after 100 cycles at $0.1 \mathrm{C}[10]$. The improved electrochemical performance of the composite was due to the fine sulfur encapsulated in polyaniline, which provided a self-breathing and flexible framework and reduced stress and structure degradation. However, uniform mixing of sulfur and polymer needs high heating temperature and long co-heating time, which may damage the polymer structure.

6 In this study, a polyaniline-wrapping hollow sulfur composite was designed and 
prepared through chemical reaction at room temperature with MCM-41 (silica) as template. The sulfur deposition amount and rate on MCM-41 surface could be regulated by the reaction of $\mathrm{Na}_{2} \mathrm{~S}_{2} \mathrm{O}_{8}$ and hydrochloric acid $(\mathrm{HCl})$. PANI was deposited on the particle surface through chemical oxidative polymerization $[11,12]$. The nano-structure of fine hollow sulfur encapsulated in polyaniline can provide many electron-conducting paths and thereby improve electrochemical performance.

\section{Experimental methods}

\subsection{Preparation of PANI-wrapping hollow sulfur}

Polyaniline-wrapping hollow sulfur composite was prepared though a three-step process. First, $0.1 \mathrm{~g}$ of MCM-41 99 wt.\% (Nan Jing Xinfeng, China), $0.091 \mathrm{~g}$ of cetyltrimethyl ammonium bromide (CTAB, analytical reagent), and $4.74 \mathrm{~g}$ of $\mathrm{Na}_{2} \mathrm{~S}_{2} \mathrm{O}_{3}$ (analytical reagent) were added to $250 \mathrm{ml}$ of deionized water, and the mixture was stirred for $5 \mathrm{~h}$. Thereafter, $100 \mathrm{ml}$ of diluted $\mathrm{HCl}$ (mass fraction of 2\%) was added dropwise to the mixture, which was stirred continuously at room temperature. Sulfur was successively deposited on the MCM-41 surface through the reaction of $\mathrm{Na}_{2} \mathrm{~S}_{2} \mathrm{O}_{3}$ and $\mathrm{HCl}$ in the following equation:

$$
2 \mathrm{HCl}+\mathrm{Na}_{2} \mathrm{~S}_{2} \mathrm{O}_{3}=2 \mathrm{NaCl}+\mathrm{H}_{2} \mathrm{O}+\mathrm{S} \downarrow+\mathrm{SO}_{2} \uparrow
$$

First, the mixture was filtered and dried to obtain S/MCM-41 composite. Second, the obtained S/MCM-41 composite, $0.091 \mathrm{~g}$ of CTAB, and $0.2 \mathrm{ml}$ of An were added to $200 \mathrm{ml}$ of diluted $\mathrm{HCl}$ (mass fraction of 3.5\%) and sonicated for $1 \mathrm{~h}$. Approximately $1 \mathrm{~g}$ of $\mathrm{Na}_{2} \mathrm{~S}_{2} \mathrm{O}_{8}(\mathrm{AR})$ was added dropwise to the mixture and stored at $0{ }^{\circ} \mathrm{C}$ with constant magnetic stirring for $3 \mathrm{~h}$. The mixture was then filtered and dried 
90 polyaniline-wrapping S/MCM-41 composite was added to the HF solution and stored

91 for $24 \mathrm{~h}$. The polyaniline-wrapping hollow sulfur composite was acquired after

96 filtration, cleaning, and drying. The detailed synthesis of the polyaniline-wrapping hollow sulfur composite is described in Fig. 1. Separate sulfur and PANI were synthesized for comparison at similar conditions.

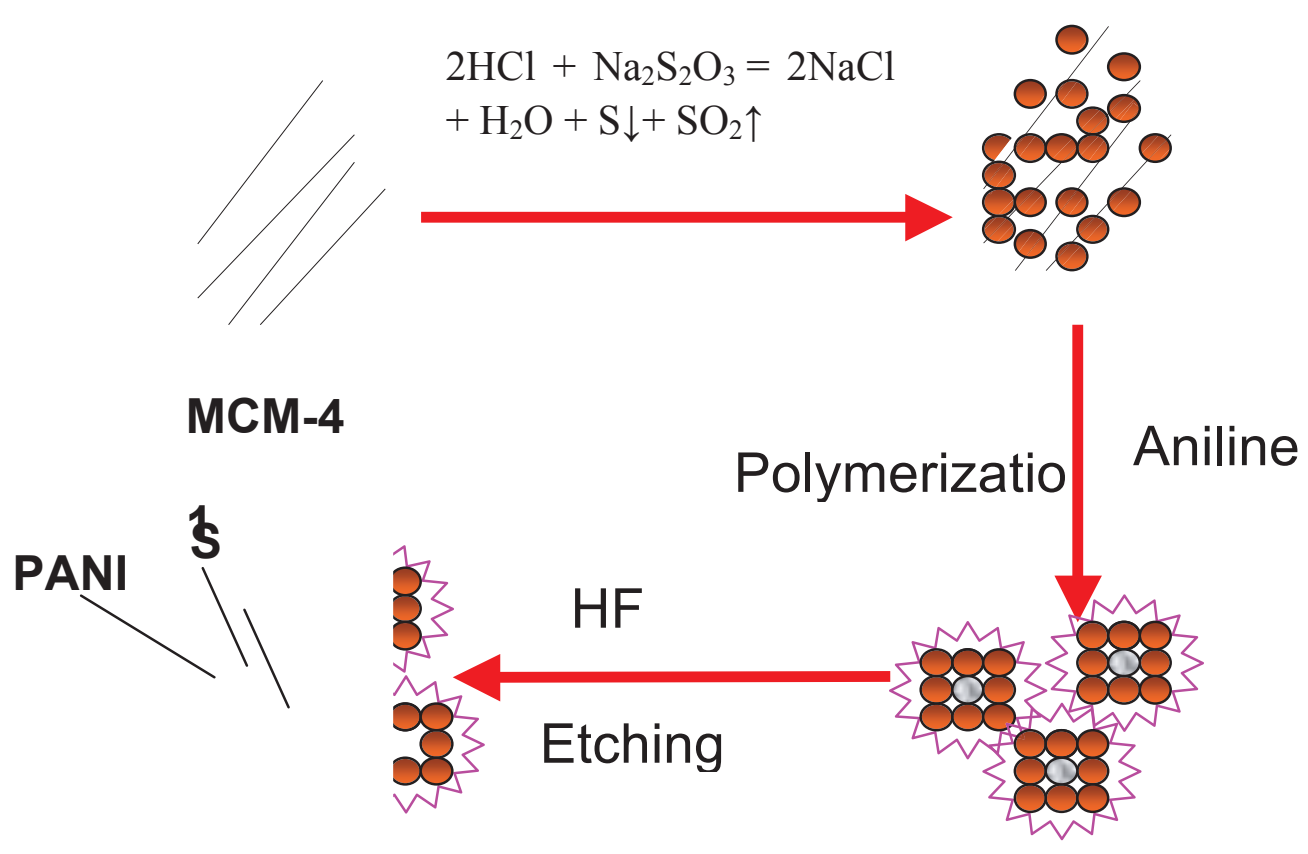

Fig. 1 Microstructure evolution of polyaniline-wrapping hollow sulfur composite in preparation process.

\subsection{Microstructure characterization}

Powder X-ray diffraction (XRD) patterns of the polyaniline-wrapping hollow sulfur composite were performed on an X-ray diffractometer (RIGAKU, Japan, model D/MAX2550V/PC). Surface morphology tests were carried out using a scanning electron microscope (JSM-5610LV) attached with EDS and transmission electron microscope (JEOL JEM-2100). The sulfur content in the polyaniline-wrapping hollow 
sulfur composite was collected using thermogravimetric analysis (METTLER Toledo SMP/PF7548) at argon atmosphere, with a temperature increase rate of $10{ }^{\circ} \mathrm{C} / \mathrm{min}$.

\subsection{Electrochemical measurements}

CR2025-type coin cells were fabricated with sulfur and polyaniline-wrapping hollow sulfur composite as the cathode and lithium as the anode. The detailed electrode preparation technology and assembly process were introduced in our previous work [13]. Cathode (80 wt.\%), acetylene black (10 wt.\%), and polyvinylidene fluoride binder (10 wt.\%) were mixed in N-methylpyrrolidinone solvent, and the mixture was stirred for $10 \mathrm{~h}$ at room temperature. The mixture was then uniformly scribbled on the surface of an aluminum foil and dried at $60{ }^{\circ} \mathrm{C}$ under vacuum for $10 \mathrm{~h}$. The coin cell was assembled in an Ar-filled glove box and was used for electrochemical characterization. In this study, $1 \mathrm{M}$ bis(trifluoromethane) sulfonamide lithium salt (Sigma Aldrich) and $0.1 \mathrm{M} \mathrm{LiNO}_{3}$ in a mixture of 1,3-dioxolane and 1,2-dimethoxyethane (volume ratio of $1: 1$ ) were used as the electrolyte.

The galvanostatic charge/discharge performance tests of the sulfur cathode were performed within the potential range of $1.5 \mathrm{~V}-3.0 \mathrm{~V}$ by using a LAND CT2001A battery-testing system at room temperature. All cells were discharged to $1.5 \mathrm{~V}$ before the test started. Specific capacities and charge/discharge current densities were calculated based on the sulfur weight in the cathode. Cyclic voltammetry (CV) and electrochemical impedance spectroscopy (EIS) were performed in the CHI660E electrochemical measurement system. CV tests were conducted with a voltage range 
143 within the frequency range of $100 \mathrm{kHz}$ to $0.01 \mathrm{~Hz}$ with an $\mathrm{AC}$ voltage amplitude of 5

$144 \mathrm{mV}$ at open-circuit voltage.

145

146

147

148 of sulfur.

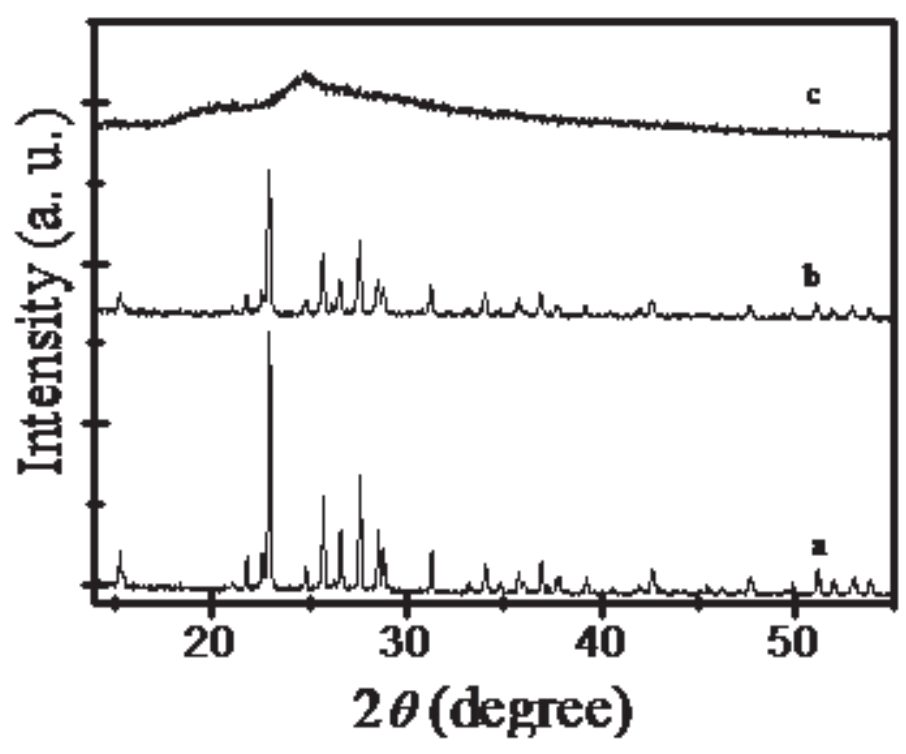

156

157

Fig. 2 XRD patterns of the sulfur (a), polyaniline wrapping hollow sulfur composite (b) and PANI(c). 

polyaniline-wrapping hollow sulfur composite were collected and shown in Fig. 3.

160 The sulfur particles are flat with irregular shapes, and the sulfur particle size ranges

161 from several micrometers to tens of micrometers, as shown in Fig. 3a. The MCM-41

162 exhibited nanofibers with several micrometers in length and tens of nanometers in 163 width and presented a hollow network structure (Fig. 3b). After sulfur was deposited 164 on the MCM-41 surface, the flat sulfur particles with several hundreds of nanometers 165 in diameter were observed (Fig. 3c). The existence of MCM-41 prevented the 166 agglomeration of sulfur particles and regulated the particle size of sulfur in the 167 preparation process. Thin nanofibers of PANI coating sulfur and numerous pores were 168 observed (Figs. 3d and 3e) after MCM-41 was etched in the HF solution, indicating 169 that polyaniline wrapped the surface of the hollow sulfur. The distribution of PANI 170 and sulfur is presented in Fig. 3e, where element $\mathrm{N}$ was uniformly distributed around 171 element S. The results confirmed that PANI was uniformly coated on the sulfur 172 surface. For further observation, transmission electron microscopy (TEM) was 173 performed to characterize the nanostructure of the polyaniline that wrapped the 174 surface of the hollow sulfur composite. Many tens of nanometers of PANI nanofibers 175 were observed on the sulfur surface, as shown in Figs. 4a and 4b, showing that fine 176 hollow sulfur was encapsulated by amorphous PANI. 


\section{0}

181

182

183

184

185

186

187

188

189

190

191

192

193

194

195

196

197

198

199

200

201 Fig.3SEM images of the sulfur (a), MCM-41 (b), S/MCM-41 (c), polyaniline

202 wrapping hollow sulfur composite (d, e) and EDS mapping in polyaniline wrapping

203 hollow sulfur composite (e). 

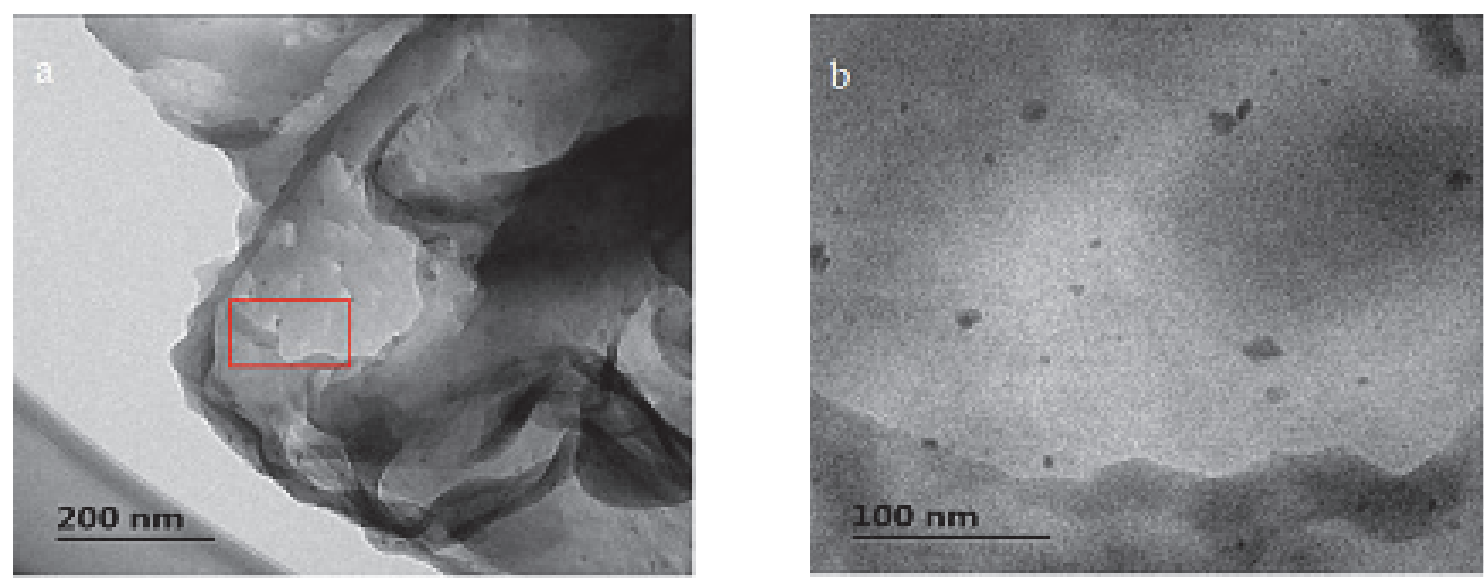

sulfur composite. Sulfur began to lose weight at $200{ }^{\circ} \mathrm{C}$ and lost approximately 100 wt.\% before reaching $320{ }^{\circ} \mathrm{C}$ (Fig. 5a). The polyaniline-wrapping hollow sulfur composite began to lose significant weight at $180{ }^{\circ} \mathrm{C}$, which is approximately $20{ }^{\circ} \mathrm{C}$ lower than that of sulfur, and presented a weight loss curve similar to that of sulfur below $285^{\circ} \mathrm{C}$. The low weight loss temperature was related to the fine hollow sulfur particles wrapped by PANI surface and was correspondingly observed in the DSC curves in Fig. 5b. Another significant difference was observed in Fig. 5b, where sulfur melting of polyaniline-wrapping hollow sulfur composite at $110^{\circ} \mathrm{C}$ was significantly lower than that of sulfur particles at $120{ }^{\circ} \mathrm{C}$. The low sulfur melting temperature stimulated sulfur diffusion through the heat generated in the charge/discharge cycles,

231 which may have a potential effect on the electrochemical performance of 232 polyaniline-wrapping hollow sulfur composite. Combined with the weight loss curve of PANI in Fig. 5a, the weight loss values of sulfur, PANI, and polyaniline-wrapping 
234 hollow sulfur composite were 100 wt.\%, 19.84 wt.\%, and 77.31 wt.\% at $320{ }^{\circ} \mathrm{C}$, 235 respectively. The sulfur content in the polyaniline-wrapping hollow sulfur composite 236 was determined to be $71.69 \%$ through the following equation:

$237 \quad(\mathrm{Ms}+\mathrm{Mp}) \times 77.31 \%=\mathrm{Ms} \times 100 \%+\mathrm{Mp} \times 19.84 \%$

238 Ms: mass reaction of sulfur in composite material

Mp: mass fraction of PANI in composite material

240
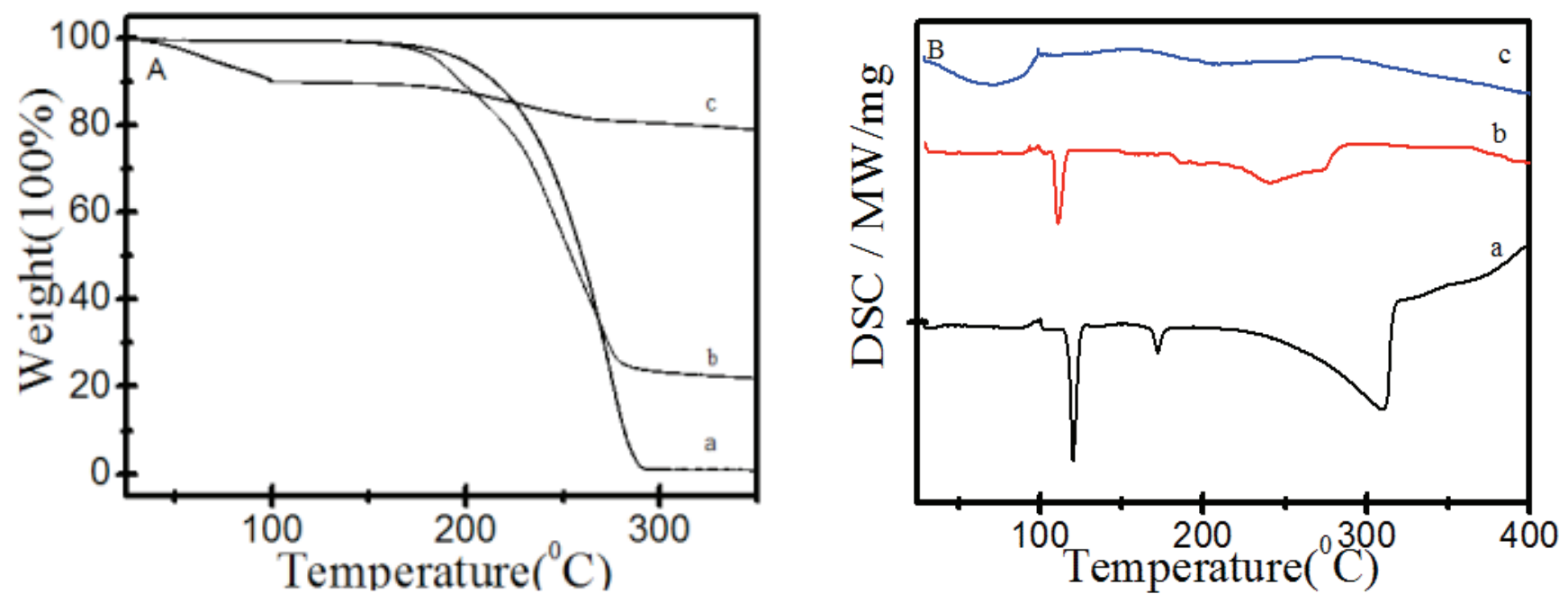

241

242 Fig. 5 TG (A) and DSC (B) curves of the sulfur (a), polyaniline wrapping hollow

243 sulfur composite (b) and PANI(c).

\subsection{Electrochemical performance}

245 Figs. $6 \mathrm{a}$ and $6 \mathrm{~b}$ show the cycle performance of sulfur and polyaniline-wrapping 246 hollow sulfur composite. The charge and discharge capacities showed an increasing 247 trend with increasing cycle time for sulfur and polyaniline-wrapping hollow sulfur 248 composite. This trend was due to the activation caused by sulfur diffusion into the 249 internal void space inside the polyaniline shell, wherein heat was generated and sulfur 
was melted during the charge/discharge process $[14,15]$. Thereafter, the charge and discharge capacities decreased when cycle time further increased. A difference was observed between sulfur and the polyaniline-wrapping hollow sulfur composite. The charge and discharge capacities of sulfur increased from its initial values of 326.7 and $268.5 \mathrm{mAh} \mathrm{g}^{-1}$ in the first cycle to the maximum values of 526 and $384.1 \mathrm{mAh} \mathrm{g}^{-1}$ at the 30 th cycle and then decreased irreversibly to 378 and $306.7 \mathrm{mAh} \mathrm{g}^{-1}$ at the 100 th cycle. The charge and discharge capacities of the polyaniline-wrapping hollow sulfur composite increased from the initial values of 526 and $31.9 \mathrm{mAh} \mathrm{g}^{-1}$ in the first cycle to the maximum values of 700.6 and $598.4 \mathrm{mAh} \mathrm{g}^{-1}$ at the 10th cycle in the activation process, 606.7 and $522.9 \mathrm{mAh} \mathrm{g}^{-1}$ at the 50th cycle, 596.6 and $513.4 \mathrm{mAh} \mathrm{g}^{-1}$ at the 100th cycle, and 593.6 and $506.3 \mathrm{mAh} \mathrm{g}^{-1}$ at the 150th cycle. The charge and discharge capacities of the polyaniline-wrapping hollow sulfur composite presented stable values after a slight decrease following the activation process, in contrast to those of sulfur.

The detailed charge and discharge curves of sulfur and polyaniline-wrapping hollow sulfur composite are shown in Figs. 6c and 6d. The sulfur exhibited no platform in the first charge and discharge curves, which corresponded to the low charge and discharge capacities. At the 30th cycle, one charge platform and two discharge platforms were observed with the highest platform length and voltage. However, the platform length and voltage worsened at the 50th and 100th cycles, accompanied by the decreased charge and discharge capacities. In contrast to the charge and discharge curves of sulfur, those of polyaniline-wrapping hollow sulfur 
composite had one good charge platform and two discharge platforms in the first cycle. The charge and discharge platform voltages of polyaniline-wrapping hollow sulfur composite maintained stable values in the entire charge and discharge cycles. However, the charge and discharge platform lengths increased and then slightly decreased from the first to the 10th, 50th, 100th and 150th cycles. The improved electrochemical performance of the polyaniline-wrapping hollow sulfur composite was related to the oxidation-reduction of the cathode in the charge/discharge process.

Figs. 6e and 6f show the $\mathrm{CV}$ curves of lithium/sulfur cells tested with $0.2 \mathrm{mV} \mathrm{s}^{-1}$ scan rate. The sulfur cathode showed two significant cathodic peaks at 1.9 and $2.3 \mathrm{~V}$ as well as one anodic peak at $2.5 \mathrm{~V}$ [16]. The cathodic peaks corresponded to the formation of short polysulfides $\left(\mathrm{S}_{6}{ }^{2-}\right.$ and $\mathrm{S}_{4}{ }^{2-}$ ) by cyclooctasulfur (S8) reduction and the subsequent formation of insoluble lithium sulfide $\left(\mathrm{Li}_{2} \mathrm{~S}_{2}\right.$ or $\left.\mathrm{Li}_{2} \mathrm{~S}\right)$ by short polysulfide reduction. In terms of the polyaniline-wrapping hollow sulfur cathode, the cathodic peak at $1.9 \mathrm{~V}$ became weak, and the peak at $2.3 \mathrm{~V}$ reduced slightly to $2.2 \mathrm{~V}$, whereas the anodic peak at $2.50 \mathrm{~V}$ increased slightly to $2.64 \mathrm{~V}$. The results reflected that the hollowed sulfur and its charge product $\mathrm{Li}_{2} \mathrm{~S}_{2}\left(\right.$ or $\left.\mathrm{Li}_{2} \mathrm{~S}\right)$ wrapped by polyaniline was easily reduced and oxidized, respectively. This finding may be due to the fact that fine hollow sulfur wrapped polyaniline had significantly more electron conduction paths and thereby improved conductivity. 

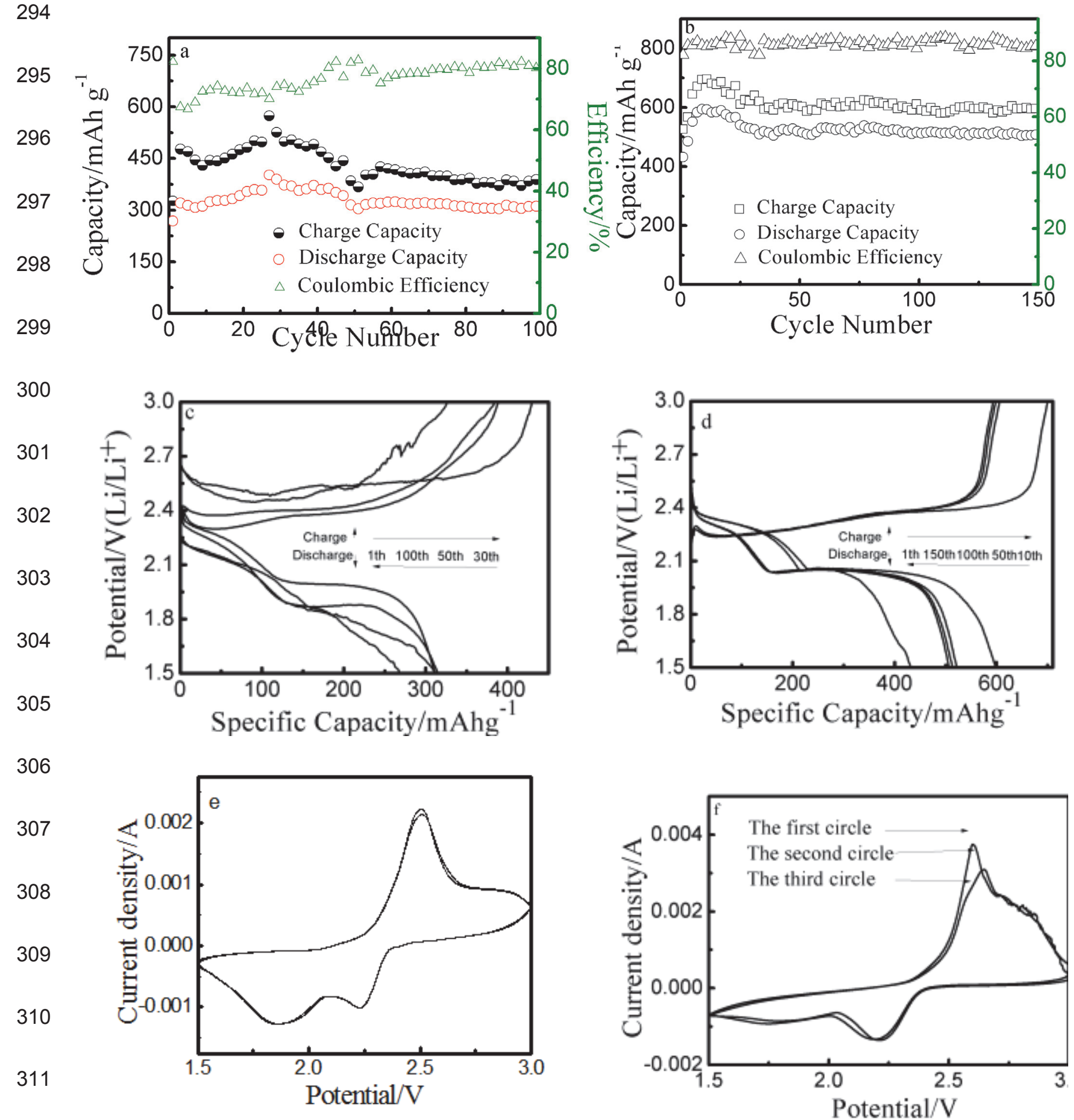

312 Fig .6 Cycle performance (a, b), charge/discharge voltage curves (c, d) and cyclic

313 voltammetry curves $(e, f)$ of sulfur and polyaniline wrapping hollow sulfur composite

314 EIS was performed to demonstrate the cycle performance improvement of the

315 polyaniline-wrapping hollow sulfur composite. Fig. 7 shows the typical Nyquist plots 
of the polyaniline-wrapping hollow sulfur composite at different charge and discharge

cycles. The Nyquist plots had a large semicircle in the high frequency regions and an

318 inclined line in the low-frequency regions before the charge and discharge cycles. The

319 semicircle was divided into two small circles after the charge and discharge cycles.

320 The semicircle at high frequency was considered as charge transfer resistances in the

321 lithium-electrolyte interface, whereas the semicircle at medium frequency was likely

322 associated with the charge transfer of lithium polysulfide intermediaries formed

323 during the charge and discharge cycles [17]. According to the EIS results at the first,

324 10th, 50th, 100th, and 150th cycles, the semicircle greatly decreased as the process

325 reached the 150th cycle, suggesting that the resistance successively reduced, and the

326 formed lithium polysulfide intermediaries in the PANI films were attributed to a high

327 conductivity for the charge transfer.

328

329

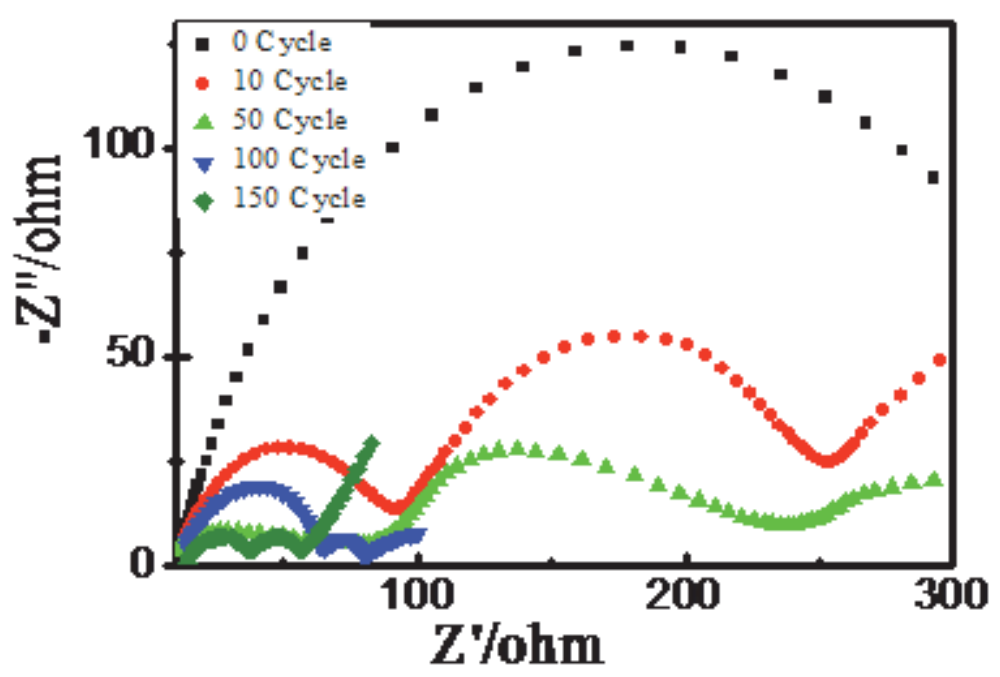

Fig. 7 Nyquist plots of polyaniline wrapping hollow sulfur composite at different cycle times.

Here, the presented paper used chemical method to prepare high-performance 
polyaniline wrapping hollow sulfur composite. The improved electrochemical performance came from hollow nanometer sulfur wrapped by polyaniline. The flexible nanstructure enhanced conductivity of sulfur and provided a large void space, which accommodated sulfur expansion and prevented polysulfide dissolution during lithiation. Compared to the conventional co-heating of sulfur and polyaniline at high temperature $[9,10]$, the chemical reactions including sulfur deposition and polyaniline wrapping occurred at room temperature and did not damage the polymer structure. The reactions could also be easily controlled to obtain fine hollow sulfur particle wrapped by polyaniline. The polyaniline wrapping hollow sulfur composite exhibited long stable cycling performance, which presented a promising future for large-scale Li-S batteries.

\section{Conclusion}

Polyaniline-wrapping hollow sulfur composite was prepared through in situ synthesis and was used to investigate the electrochemical properties of lithium/sulfur cells. The polyaniline-wrapping hollow sulfur composite presented an excellent reversible capacity of $506.3 \mathrm{mAh} \mathrm{g}^{-1}$ after 150 cycles at $0.1 \mathrm{C}$, which was significantly higher than that of sulfur at similar conditions. Microstructure and electrochemical results showed that the capacity increase was related to the following factors: increase of sulfur utilization because of a smaller sulfur particle size, enhancement of electrical contact, and decrease of active material loss because of sulfur and its charge product $\mathrm{Li}_{2} \mathrm{~S}_{2}$ (or $\mathrm{Li}_{2} \mathrm{~S}$ ) wrapped by polyaniline.

\section{Acknowledgments}


354 355 356 357 358 359 360 361 362 363 364 365 366

This work was financially supported by the Scientific Research Foundation for the Returned Scholars, postdoctoral support of P. R China (2015M581910), postdoctoral preferential support of Zhejiang province (BSH1502029), the National Science Foundation of China (Project No. 51501175), and the Guangxi Key Laboratory of Information Materials (Guilin University of Electronic Technology, project No. 1210908-02-K).

\section{Reference}

[1] Shim J., Striebel K. A., Cairns E. J. The lithium/sulfur rechargeable cell. J. Electrochem. SoC., 2002, 149: a1321-a1325.

[2] Chen S., Huang X., Liu H. 3D Hyperbranched Hollow Carbon Nanorod Architectures for High-Performance Lithium-Sulfur Batteries [J]. Advanced Energy Materials, 2014, 4(8): 1301761.

[3] Yamin H., Peled E. Electrochemistry of a nonaqueous lithium/sulfur rechargeable cell. J. Power sources, 1983, 9:281-287.

[4] Yin Y. X., Xin S., Guo Y. G., Wan L. J. L.ithium-Sulfur Batteries: Electrochemistry, Materials, and Prospects. Angew. Chem. Int. Edit., 2013, 52: 13186-13200.

[5] Bresser D., Passerini S., Scrosati B. Recent process and remaining challenges in sulfur-based lithium secondary batteries-a review. Chem. Commun., 2013, 49:10545-10562.

[6] Zhou W. D., Yu Y. C., Chen H., Disalvo F. J., Abruna H. D. Yolk-shell structure of polyaniline-coated sulfur for thium-sulfur batteries. J Am Chem Soc, 2013, 135(44):16736-16743. 
[7] Li W. Y., Zhang Q. F., Zheng G. Y., She Z. W., Yao H. B., Cui Y. Understanding the role of different conductive polymers in improving the nanostructure sulfur cathode performance. Nano Lett., 2013, 13(11): 5534-5540.

[8] Wang L. Chen J., Konstantinov K., Zhao L., Ng S. H., Wang G. X., Guo Z. P., Liu H. K. Sulphur-polypyrrole composite positive electrode materials for rechargeable lithium batteries. Electrochemica Acta, 2006, 51(22): 4634-4638.

[9] Qiu L.L., Zhang S.C., Zhang L., Sun M. M., Wang W.K.. Preparation and enhanced electrochemical properties of nano-sulfur/poly (pyrrole-aniline) cathode material for lithium/sulfur batteries. Electrochemica Acta, 2010, 55, 4632-4636.

[10] Xiao L., Cao Y., Schwenzer B., Engelhard M.H., Saraf L. V., Nie Z., Exarhos G.J., Liu J. A solt approach to encapsulate sulfur: polyaniline nanotubes for lithium-sulfur batteries with long cycle life. Adv. Mater., 2012, 24, 1176-1181.

[11] Ma G. Q., Wen Z. Y., Jin J., Lu Y., Wu X. W., Wu M. F., Chen C.H. Hollow polyaniline sphere@sulfur composites for prolonged cycling stability of lithium-sulfur batteries. J. Materials Chemistry A, 2014, 2: 10350-10354.

[12] Wang M. Q., Wang W. K., Wang A. B., Yuan K. Q., Miao L. M., Zhang X. L., Huang Y. Q., Yu Z. B., Qiu J. Q. Amulti-core-shell structured composite cathode material with a conductive polymer network for Li-S batteries. Chem. Commun., 2013, 49, 10263-10265.

[13] Wei P., Fan M. Q., Chen H. C., Yang X. R., Wu H. M., Chen J. D., Li T., Zeng L. W., Zou Y. J. High-capacity grapheme/sulfur/polyaniline ternary composite cathode with stable cycling performance. Electrochemica Acta, 2015, 174: 963-969. 
399 [14] Ryu H. S., Ahn H. J., Kim K. W., Ahn J. H., Lee J. Y. Discharge process of $400 \mathrm{Li} / \mathrm{PVdF} / \mathrm{S}$ cells at room temperature. J. Power Sources, 2006, 153:360-364.

401 [15] Wang D. W., Zeng Q. C., Zhou G. M., Yin L. C., Li F., Cheng H. M., Gentle I. 402 R.. Carbon-sulfur composites for Li-S batteries: status and prospects. J. Mater. Chem., $4032013,1,9382-9394$.

404 [16] Choi Y.J., Chung Y.D., Baek C.Y., Kim K.W. Effects of carbon coating on the 405 electrochemical properties of sulfur cathode for lithium/sulfur cell. J Power Sources, $406 \quad 2008,184: 548-552$.

407 [17] Konarov A., Gosselink D., Doan T. N. L., Zhang Y. G., Chen P. Simple, scalable, 408 and economical preparation of sulfur- PANI composite cathode for Li/S batteries. J. 409 Power Sources, 2014, 259: 183-187. 